\title{
Radiomics in Head and \\ Neck Cancers Radiotherapy. Promises and Challenges
}

Roxana Irina IANCU ${ }^{a, b}$, A. D. ZARA ${ }^{c, d}$, C. C. MIRESTEANe, f, D. P. T. IANCU ${ }^{a, c}$

a"Gr. T. Popa" University of Medicine and Pharmacy, lasi, Romania

b"St. Spiridon" Emergency Hospital, Iaşi, Romania

'Regional Institute of Oncology, lasi, Romania

d“Alexandru loan Cuza" University, lasi, Romania

eUniversity of Medicine and Pharmacy of Craiova, Craiova, Romania

fRailways Clinical Hospital, lasi, Romania

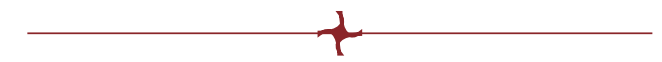

\begin{abstract}
Radiomics, a subdomain of artificial intelligence, consists in extracting a large volume of data from all medical imaging techniques and correlating them with clinical data in order to build predictive and prognostic models. Radiomics is related to radiogenomics that correlates genetic mutations and molecular and biological characteristics of tissues with information extracted from medical imaging. Both are state-of-the-art fields of translational biomedical research. The ability to predict early patient survival and response to treatment, but also the capacity to identify tumor subtypes non-invasively, could make radiomics a key player with an essential role in personalized oncology. In head and neck cancer radiotherapy, radiomic algorithms can predict not only the response to radiochemotherapy or induction chemotherapy but also the need for planning through adaptive radiotherapy (ART). Radiomics can also predict the risk of severe toxicities, especially that of xerostomia. Given the benefit that a de-escalation of treatment can bring in selected cases to improve the quality of life, radiomics is expected to be part of the therapeutic decision for head and neck cancers in the near future, and may help identify cases where de-escalation of multimodal therapy will not jeopardize the therapeutic benefit.
\end{abstract}

Keywords: radiomics, radiogenomics, radiotherapy, head and neck cancers, oncology. 


\section{INTRODUCTION}

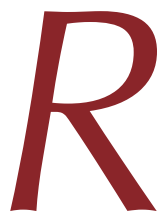
adiomics is a modern and innovative method, derived from artificial intelligence (AI), and consists in extracting large amounts of quantitative features from digital medical images. Radiomics analysis can be performed using high-resolution structural imaging such as computed tomography $(\mathrm{CT})$, magnetic resonance imaging (MRI), but also from digital radiography (RD) or ultrasonography (US). Hybrid imaging, and especially positron emission tomography computed tomography (PET-CT), is also used to extract radiomic features. Subsequently, these features are correlated with clinical and biological data to build predictive and prognostic models with clinical utility. The number of articles proposing radiomic analysis in all areas of clinical medicine to aid in medical decision-making is growing. In oncology, the correlation of mining data from malignant tumor images in order to "decode" them is a constantly expanding research direction. The growing need for biomarkers in the context of stratification of oncological disease turns radiomics into a key player in precision oncology. Radomics as "medical imaging biomarker" could be useful to predict the risk of recurrence after surgery and the response and duration of response either post-radiotherapy treatment or after systemic oncological treatments (chemotherapy, target molecular therapy, immunotherapy). The possibility of predicting non-invasively and with minimal data costs obtained by genomics and proteomics techniques is the object of the rule called "radiogenomics" $(1,2)$.

\section{The five steps of radiomics}

In most cases, radiomic analysis consists of five distinct stages: image aquisition, segmentation, features extraction, feature selection and radiomic analysis. Here, we only consider the traditional approach in radiomics, and not deep learning, the modern method based on the concept of neural networks that is used to stimulate human brain behavior by employing multi-layer learning for the analysis of medical images.

Acquisition of medical images is the first and also an essential stage, being an important source of uncertainties whenever certain standardiza- tion rules are not implemented. A higher resolution aquisition and protocol standardization are essential in obtaining scientifically valid results. It is recommended that image acquisition should be done on the same machine and with the same acquisition protocol and parameters, whether we consider images obtained from ultrasonography, CT, MRI or PET-CT. Data preparation consists in selecting images that respect a protocol as standardized as being reproducible, eliminating those that do not meet the standards imposed in the study. In this stage, the type of target volume chosen (primary tumor, lymph node or distant metastasis) will also be taken into account, with either just one category or all categories being selected. The stage of data preparation and filtering also includes the choice of a final objective that will be the object of correlations (general survival, recurrence, complete or partial response, tumor progression).

The next step, segmentation, aims to determine the volume of interest (VOI) and to delineate it. Given the subjectivity in assessing the margins and delineation of a tumor volume by an expert, the option of choosing an automatic delineation algorithm or delineation is made by two experts in order to limit interobserver variability. In terms of standardization, automatic segmentation based on identification of standardized voxel values is preferred with respect to reproducibility. However, if the PET-CT technique was used, automatic self-segmentation without supervision of a nuclear medicine expert could lead to the identification of normal metabolically active tissues such as tumors.

Feature extraction is the stage in which a software application is used to extract data from VOI. In the radiomic analysis algorithm, the set of images is generally divided into two subsets: a model training set that includes about $80 \%$ of images and a validation set that includes the rest of the medical images. In this stage, we can extract first order features, which include the distribution of pixel intensity, shape features, and texture features, which include data regarding the matrix of gray levels. Texture features are an imagistic correspondence of tumor heterogeneity. Wavelet features are more difficult intuitive features that describe textural and intensity features extracted from images using a wavelet decompostion algorithm. Free software applications 
such as IBEX, LIFEx or MaZda radiomics can be used to extract these features.

Feature selection is a process of reducing the number of variables in order to build a model that is as valid and less expensive as possible. The choice of features can be an automatic process, by either a statistical selection of those characteristics which are correlated with the clinical endpoint or manual selection according to the literature data. Thus, the radiomic process can be supervised or unsupervised.

The creation of the radiomic model involves the association of some characteristics or sets of characteristics with clinical data but also with molecular and genetic mutations characteristic of tumors and tumor tissues. Thus, both radiomics and radiogenomics become non-invasive techniques to support the diagnosis and to predict some variables involved in the therapeutic decision. Algorithms such as vector machine support (SVM) or random forest are used to create a radio signature that will include only those features that are correlated with the evaluated characteristics. SVM is a learning algorithm (3-5) (Figure 1).

\section{Radiomics and radiotherapy/chemotherapy/ radiochemotherapy for HNC}

Head and neck cancers, especially squamous cell carcinoma of the head and neck (HNSCC), are the sixth most common cancer worldwide, with the number of newly diagnosed cases being about 650,000 per year. In the locally advanced stages, the non-surgical treatment that offers the possibility of organ preservation is considered a therapeutic standard, consisting of radiotherapy and chemotherapy, the biggest challenge being to obtain the optimal treatment with limited toxicity. High-precision radiotheray techniques, 3D-conformal (3D-CRT), intensity-modulated radiotherapy (IMRT) and intensity-modulated volumetric arc therapy (VMAT) are currently used to optimally conform the tumor target volumes to the prescribed radiation dose, thus protecting normal tissues and radiosensitive anatomical structures in the vicinity. The concept of image-guided radiotherapy (IGRT) is the basis for obtaining a ballistic precision, essential for accurately identifying and irradiation of target volumes and for radiosensitive structures sparing. If CT simulation became a standard in the IGRT era, the use of MRI and PET-CT helps to more accurate identification of tumor margins, but also to a metabolic characterization of cancer, with therapeutic and evolutionary implications. The concept of adaptive radiotherapy (ART) involves treatment replanning after a number of delivered radiation fractions in order to respond to anatomical changes in the irradiated region or

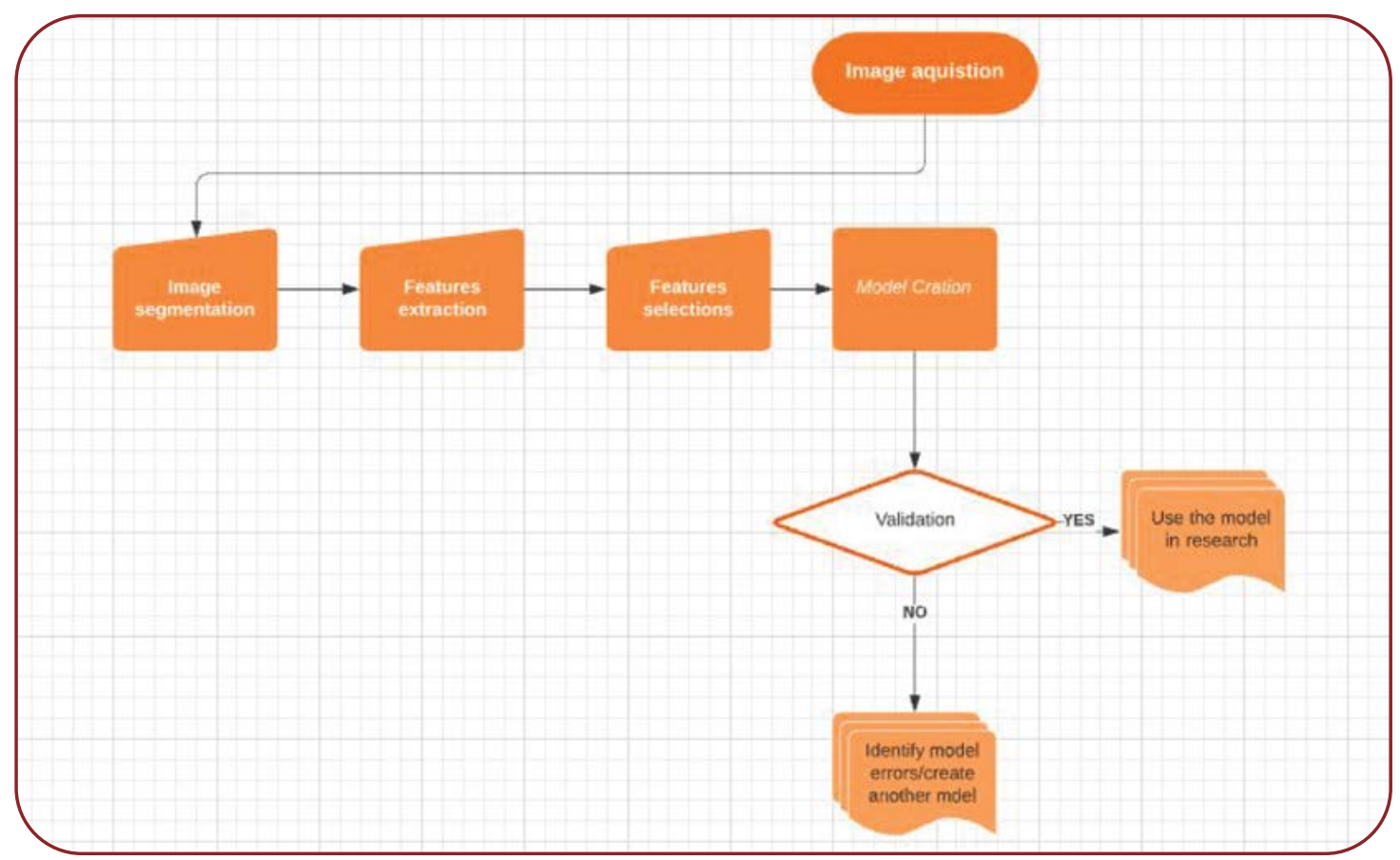

FIGURE 1. Radiomic analysis algorithm 
to change the prescription dose and fractionation regimen according to the biological response of the tumor to irradiation $(6,7)$.

Although there have been improvements in both the quality of HNC radiotherapy and overall survival (OS) rate at five years has improved by less than $15 \%$ from $52.7 \%$ to $65.9 \%$ in the last two decades of the $20^{\text {th }}$ century. The change in regional anatomy during the 6-7 weeks of treatment is one of the causes that can affect the accuracy of treatment with possible implications in the therapeutic ration. Reductions in the size of the target volume (the primary tumor or lymph nodes) and parotid glands shrinkage are known to occur during radiotherapy, affecting the dosimetry, but also increasing the risk of planning target volumes (PTVs) "geographic miss". Ballistic errors due to changes in the anatomy of structures can occur in case of irradiation or could be asociated with high dose irradiation of organs at risk (OARs). Adaptive radiotherapy (ART) is a concept precisely developed in order to minimize these anatomical variations and their clinical implications. In fact, the concept includes two distinct trends: adapted anatomy (A-ART), where the prescribed doses on target volumes remain the same but only the regional anatomy changes, and adapted ART response (R-ART), a concept based on new radiosensitivity data and on re-evaluation of doses delivered in volumes or subvolumes for the purpose of escalation in regions of radioresistance in order to improve tumor control and de-escalation in regions with increased radiosensitivity, in order to reduce the risk of treatment-associated toxicities $(8,9)$.

The use of medical imaging as a standard in both diagnosis and evaluation of treatment response as well as in the planning of radiotherapy treatment opens new opportunities for radiomic analysis to improve HNC management. In the last decade, conventional radiological imaging has become not only the object of study of the imaging physician but also a "mine" from which large amounts of data can be extracted. Having the capacity to extract information about tumor heterogeneity using data mining, radiomics is a key player in precision medicine. However, the use of radiomics in clinical practice can currently only be used to guide the clinician's decision, without being implemented as a validated method, one of the reasons being the impossibility of standardization, a consensus in data acquisi- tion, processing and reporting being urgently needed. Analyzing the capabilities offered by radiomics, it is easy to anticipate possible applications of the method in the multimodal therapeutic decision of HNC but also for radiotherapy treatment planning. From a predictive of the response to radiotherapy or radiochemotherapy point of view, radiomics can non-invasively anticipate the effect of treatment at established time intervals. Radiomics features range from simple and easy to intuit, like "mean”, "shape” and "volume", to more complex such as "gray level co-occurence matrix" or "kurtosis", which are more difficult to understand and more "abstract". Especially for the particular case of radiotherapy, the segmentation of tumors for radiomatic analysis can be assimilated with delineation of target volumes. Shape, volume, sphericity are geometric features that can be extracted from the treatment planning systems (TPS) platforms. Hounsfield units (HUs), a relative quantitative measurement of density used in CT imaging or standard absorption values (SUVs) used to quantify tumor metabolism in PET, are currently standardized as units of quantification in imaging but represent only some of the data that can be mined from high resolution images $(10,11)$.

Concerns to improve the performance of radiotherapy have also focused on the field of radiobiology, and more precisely, radiosensitivity. The involvement of genetic mutations in intrinsic radiosensitivity is demonstrated by the introduction of the radiosensitivity index (RSI) in the evaluation of tumor radiosensitivity. Reducing the genetic panel involved in radiosensitivity, proposed by Eschrich et al at 10 RSI genes, becomes a tool with potential for clinical application. The model of adjustment of the LQ quadratic linear model, proposed by Scott et al, genomically adjusted radiation dose (GRAD) may also contribute to the personalization of radiotherapy and individualization of standard 2 Gy fractions. Thus, radiogenomics opens new horizons in predicting non-invasive tumor radiosensitivity. Radiogenomics and radiomics were investigated in $\mathrm{HNC}$, demonstrating the ability to predict pathological features, nodal and extranodal metastases, HPV status, toxicity of treatmets. Xerostomy, trismus and hearing loss were toxicities of radiotherapy or radiochemotherapy analyzed in radiomic studies on patients irradiated with HNC. Mutations such as TP53 CDKN2A, and PI3k, 
which are commonly found in HNC, methylations of tumor suppressor genes such as RARB, DCC and MGMT, that may have downregulated expressions with clinical implications, have also been analyzed by radiomics (12-15).

Radiomics has demonstrated the ability to predict a tumor subtype of HNC characterized by human papillomavirus (HPV) status, tumor subtype of major importance especially in the case of oropharyngeal cancer, HPV status being included in the new TNM staging. With a reported increase in the prevalence of from $50.7 \%$ at the end of the last millennium to $69.7 \%$ in the first 10 years of the new millennium in North America, oropharyngeal cancers associated with HPV infection are characterized by major prognostic and response to therapy differences compared to ENT cancers associated with tobacco use. Radiomics prediction of the HPV etiology involvement in $\mathrm{HNC}$ is one of the most studied applications. The p16 immuno-histochemical evaluation, considered a surrogate biomarker, was frequently correlated with radiomics features extracted from CT images and less frequently from PET-CT and MRI. The image selection algorithm was different across various studies, the use of contrast agent being one of the variables. Texture features were most commonly correlated with HPV status for HNC, especially for oropharyngeal cancers. The choice of VOI in the metastatic lymph node and the extraction of radiomics features from MRI and PET-CT medical imaging are still less studied and will have to be validated as methods for radiomic evaluation of $\operatorname{HNC}(16,17)$.

Zhu et al's radiomic study was not limited only to the identification of HPV status, but the analysis was also associating the TP53 mutation detection. The authors identified two biomarkers with potential of prognostic for HNC by using a random forest classification algorithm. The radiomic model proposed has also proven the ability to early predict the response to radiotherapy and chemotherapy. From two independent HNC cohorts, a training cohort of 101 cases and a validation cohort including 95 patients, 440 radiomic features were extracted. Subsequently a prognostic models were created using three classifiers. The authors identified the type of the chosen classifier as a factor associated with predictive performance. The study estimates that radiomics will provide precision imaging bio- markers for HNC patients in the future. Analyzing pre-treatment $\mathrm{CT}$ images without contrast agent, from 465 patients with oropharyngeal cancer, Head and Neck Quantitative Imaging Working Group of M.D. Anderson Cancer Center proposes a two-feature radiomic signature, coonsidered with a higher predictive power. Most of the studies proposed multivariate Cox proportional hazard models and the three options regarding the prefered number and association of radiomic features are as follows: „single feature", ,signature" or "radiomic score" $(18,19)$.

Errors and uncertainties in the reproducibility of radiomic studies may be present at all stages of the radiomic process. From the point of view of radiomic features, first order features, and especially entropy, are the most reproducible. Welch et al propose a set of safeguards in order to improve the radiomic signature by identifying vulnerabilities in the development of radiomic signature. The authors proposed external validation of a radiomic model (MW2018) by comparison with radiomic data obtained from HNC tumors and lung cancer (20-22).

The value of radiomic analysis for radiotherapy planning has been demonstrated not only in the case of HNC. Automatic segmentation of organs at risk (OARs) is based on Al algorithms and greatly facilitates the activity of radiotherapy planning. While training a radiomic model using images from $15 \mathrm{HNC}$ patients aquired by pretreatment and post-treatment MRI, Yang et al proposed a model for automatic contouring of the parotid glands. The radiomic model can be implemented in the process automation for changes in the shape and size of the parotid glands (shrinkage) during radiotherapy treatment. The development of predictive models in order to predict tumor control probability (TCP) before treatment and to assess the toxicity risk, quantified by using normal tissue complication probability (NTCP), is based on dosimetric data extracted from dose volume histograms (DVH). Toxicities with the potential to severely affect the quality of life (QoL), even causing treatment discontinuation, such as dysphagia, mucositis and xerostomia, are included in NTCP models.

Retrospectively analyzing CT, MRI, and clinical data including toxicities, evalauated according to The Common Terminology Criteria for Adverse Events (CTACAE) from 266 patients treated with radiotherapy between 2009 and 
2018 for HNC, Sheikh and collaborators designed a radiomic model in order to predict treatment-associated xerostomia. The parameters extracted from the DVH were evaluated too. Spearman and Lasso regression correlation was used in statistical analysis and selection of radiomic characteristics. Analyzing comparatively eight models that included combined CT, MRI, $\mathrm{DVH}$ and clinical data, the authors concluded that radiomic analysis of CT and MRI images could be used in predicting xerostomia for $\mathrm{HNC}$ patients tretead with radiotherapy or radiochemotherapy. ART is a topic of interest especially in the case of HNSCC through the horizons offered to reduce toxicities particularly in patients whose anatomy varies during treatment. The concept includes two directions, one in which treatment rescheduling is done only based on the anatomical change, and another one in which the fractionation protocol is adjusted by escalation and de-escalation depending on the biological/clinical response to treatment. In order to identify tumor radiomic biomarkers for early prediction of ART planning necessity, data from 70 patients with nasopharyngeal cancer were extracted from pre-treatment MRI images. From the IMR T1-w and T2-w contrast images processed and filtered with Laplacian of Gaussian algorithm, 479 first-order radiomic characteristics, texture and shape were extracted. LASSO regression was used to build the model and Area Under The Curve (AUC) - Receiver Operating Characteristics (ROC) was used to test the model performance. The model predicted the need for ART, with 13 patients out of 70 benefiting from an adaptive planning protocol. The authors of a randomized phase II study evaluating the benefit of a consolidation protocol with cetuximab after concurrent radiochemotherapy + cetuximab (triplet), in patients with locallyadvanced $\mathrm{HNC}$, have also performed a radiomic analysis to identify a correlation between serum biomarkers and radiomics features. The authors propose a model based on the concept that higher entropy and the complexity of Hounsfield units distribution in tumor tissue could predict both local and regional (nodal) control (23-25).

\section{CONCLUSIONS}

adiomics is one of the most challenging areas of Al application in medicine. Through the ability to predict survival and treatment response but also to identify non-invasive tumor subtypes, radiomics could play an essential role in personalized oncology. In head and neck cancer radiotherapy, radiomic algorithms can predict not only the response to radiochemotherapy or induction chemotherapy, but also the need for planning through adaptive radiotherapy (ART). Radiomics can also predict the risk of severe toxicities, especially that of xerostomia. Radiomics is expected to be implemented in the clinical and dosimetric decision of radiotherapy planning for $\mathrm{HNC}$, one of the applications of interest being the identification of patients candidates for de-escalation of treatment, with the ultimate goal of improving their quality of life by limiting toxicity.

Conflicts of interest: none declared.

Financial support: none declared.

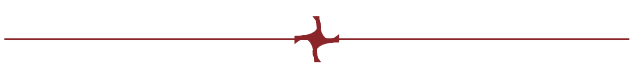

\section{R}

1. Liu Z, Wang S, Dong D, et al. The Applications of Radiomics in Precision Diagnosis and Treatment of Oncology: Opportunities and Challenges. Theranostics 2019;9:1303-1322. Published 2019 Feb 12. doi:10.7150/thno.30309.

2. Lo Gullo R, Daimiel I, Morris EA, Pinker K. Combining molecular and imaging metrics in cancer: radiogenomics. Insights Imaging 2020;11:1.

3. Bogowicz M, Vuong D, Huellner MW, et al. CT radiomics and PET radiomics: ready for clinical implementation?
Q J Nucl Med Mol Imaging 2019;63:355-370.

4. Lambin P, Leijenaar RTH, Deist TM, et al. Radiomics: the bridge between medical imaging and personalized medicine.

Nat Rev Clin Oncol 2017;14:749-762. doi: 10.1038/nrclinonc.2017.141.

5. Mehanna $H$, Beech $T$, Nicholson $T$, et al. Prevalence of human papillomavirus in oropharyngeal and nonoropharyngeal head and neck cancer--systematic review and meta-analysis of trends by time and region. Head Neck 2013;35:747-755.
6. Feng $M$, Eisbruch A. Future issues in highly conformal radiotherapy for head and neck cancer.

J Clin Oncol 2007;25:1009-1013. doi: 10.1200/JCO.2006.10.4638.

7. Castelli J, Simon A, Lafond C, et al. Adaptive radiotherapy for head and neck cancer. Acta Oncol 2018;57:1284-1292.

8. Pulte D, Brenner H. Changes in survival in head and neck cancers in the late $20 \mathrm{t}^{\mathrm{h}}$ and early $21^{\text {st }}$ century: a period analysis. Oncologist 2010;15:994-1001. doi:10.1634/theoncologist.2009-0289. 
9. Morgan HE, Sher DJ. Adaptive radiotherapy for head and neck cancer. Cancers Head Neck 2020;5:1.

10. Gardin I, Grégoire V, Gibon D, et al. Radiomics: Principles and radiotherapy applications. Crit Rev Oncol Hematol 2019;138:44-50.

11. Wang C, Li H, Jiaerken $Y$, et al. Building CT Radiomics-Based Models for Preoperatively Predicting Malignant Potential and Mitotic Count of Gastrointestinal Stromal Tumors. Transl Oncol 2019;12:1229-1236. doi: 10.1016/j.tranon.2019.06.005.

12. Bruixola G, Remacha E, Jiménez-Pastor $\mathbf{A}$, et al. Radiomics and radiogenomics in head and neck squamous cell carcinoma: Potential contribution to patient management and challenges. Cancer Treat Rev 2021;99:102263.

13. Grass GD, Mills MN, Scott JG, et al. Genomics and radiomics: Tools to see the unseen to personalize radiation therapy. Appl Rad Oncol 2019;8:9-22.

14. Eschrich SA, Pramana J, Zhang $\mathrm{H}$, et al. A gene expression model of intrinsic tumor radiosensitivity: prediction of response and prognosis after chemoradiation. Int J Radiat Oncol Biol Phys 2009;75:489-496.
15. Scott JG, Berglund A, Schell MJ, et al. A genome-based model for adjusting radiotherapy dose (GARD): a retrospective, cohort-based study. Lancet Oncol 2016;18:202-211.

16. Buch K, Fujita A, Li B, et al. Using texture analysis to determine human papillomavirus status of Oropharyngeal squamous cell carcinomas on CT. AJNR Am J Neuroradiol 2015;36:1343-1348.

17. Parmar C, Grossmann P, Rietveld D, et al. Radiomic Machine-Learning Classifiers for Prognostic Biomarkers of Head and Neck Cancer. Front Oncol 2015;5:272.

18. Guha A, Connor S, Anjari M, et al. Radiomic analysis for response assessment in advanced head and neck cancers, a distant dream or an inevitable reality? A systematic review of the current level of evidence. Br J Radiol 2019;93:20190496.

19. Yang $X, W u ~ N$, Cheng G, et al. Automated segmentation of the parotid gland based on atlas registration and machine learning: a longitudinal MRI study in head-and-neck radiation therapy.

Int J Radiat Oncol Biol Phys

2014;90:1225-1233.

10.1016/j.ijrobp.2014.08.350.
20. Traverso A, Wee L, Dekker A, Gillies R. Repeatability and Reproducibility of Radiomic Features: A Systematic Review. Int J Radiat Oncol Biol Phys 2018;102:1143-1158.

21. Welch ML, McIntosh C, Haibe-Kains B, et al. Vulnerabilities of radiomic signature development: The need for safeguards. Radiother Oncol 2019;130:2-9.

22. Lohmann $P$, Bousabarah $K$, Hoevels $M$, et al. Radiomics in radiation oncologybasics, methods, and limitations. Strahlenther Onkol 2020;196:848-855.

23. Riesterer O, Pruschy M, Bender S, et al. Consolidation cetuximab after concurrent triplet radiochemotherapy+cetuximab in patients with advanced head and neck cancer: A randomized phase II study. Radiother Oncol 2020;150:62-69.

24. Sheikh K, Lee SH, Cheng Z, et al. Predicting acute radiation induced xerostomia in head and neck Cancer using MR and CT Radiomics of parotid and submandibular glands. Radiat Oncol 2019;14:131.

25. Yu TT, Lam SK, To LH, et al. Pretreatment Prediction of Adaptive Radiation Therapy Eligibility Using MRI-Based Radiomics for Advanced Nasopharyngeal Carcinoma Patients. Front Oncol 2019;9:1050. 